\title{
Gain in p-doped quantum dot lasers
}

\author{
P. M. Smowton ${ }^{\text {a) }}$ and I. C. Sandall \\ School of Physics and Astronomy, Cardiff University, Cardiff CF24 3AA, United Kingdom \\ H. Y. Liu and M. Hopkinson \\ EPSRC National Centre for III-V Technologies, University of Sheffield, Sheffield S1 3JD, United Kingdom
}

(Received 26 April 2006; accepted 27 October 2006; published online 10 January 2007)

\begin{abstract}
We directly measure the gain and threshold characteristics of three quantum dot laser structures that are identical except for the level of modulation doping. The maximum modal gain increases at fixed quasi-Fermi level separation as the nominal number of acceptors increases from 0 to 15 to 50 per dot. These results are consistent with a simple model where the available electrons and holes are distributed over the dot, wetting layer, and quantum well states according to Fermi-Dirac statistics. The nonradiative recombination rate at fixed quasi-Fermi level separation is also higher for the $p$-doped samples leading to little increase in the gain that can be achieved at a fixed current density. However, we demonstrate that in other similar samples, where the difference in the measured nonradiative recombination is less pronounced, $p$ doping can lead to a higher modal gain at a fixed current density. (C) 2007 American Institute of Physics. [DOI: 10.1063/1.2405738]
\end{abstract}

\section{INTRODUCTION}

The serious asymmetry in the movement of the electron and hole quasi-Fermi levels into the conduction and valence bands and the resulting increase in the carrier density required to reach transparency for III-V materials were described by Yablonovitch and Kane ${ }^{1}$ in 1986 in their seminal paper on how the application of strain could improve the performance of semiconductor lasers. In the case of bulk and quantum well $(\mathrm{QW})$ materials the asymmetry in the movement of the quasi-Fermi levels arises due to the asymmetry in the density of states (DOS) in the conduction and valence bands. The application of either compressive or tensile strain to make the conduction and uppermost valence bands more symmetrical and hence the movement of the quasi-Fermi levels more symmetrical reduces the carrier density necessary to reach transparency and leads to the lowest threshold current density semiconductor lasers ${ }^{2,3}$ [before the advent of quantum dot (QD) lasers].

In QD lasers it was originally thought that the use of a single electron and hole to reach transparency in a pair of states, with the implication of a symmetrical movement of the quasi-Fermi levels, would lead to ideal laser performance, but this has not yet been realized due to the requirement for an ensemble of states to be present to generate sufficient gain to overcome threshold losses. In addition, due to the much larger valence mass in III-V materials the allowed hole states in the quantum dot potential well are much more closely spaced, in energy, than the corresponding conduction states, and along with the asymmetry in the wetting layer states this leads to a large asymmetry in the movement of the quasi-Fermi levels. In quantum dot materials it is not as easy to engineer the level of strain to counter these effects as in quantum wells due to the nature of the self-assembly process.

In 1988 Vahala and $\mathrm{Zah}^{4}$ suggested that the application

${ }^{a)}$ Electronic mail: smowtonpm@cf.ac.uk of $p$ doping to a $\mathrm{QW}$ could reduce the asymmetry in the distribution of the quasi-Fermi levels. Since both the electron and hole states can be fully populated, gain and differential gain are both maximized. The application of $p$ doping in QW lasers to reduce the threshold current has not been widely employed due to problems such as an increase in the internal optical mode loss and an increase in nonradiative recombination. However, it was quickly realized that the application of modulation doping to quantum dots would be beneficial ${ }^{5}$ since a much lower level of $p$ doping should be necessary in dot material due to the lower number of allowed states, which may circumvent some of the problems seen with QW devices.

Self-assembled In(Ga)As QD lasers using $p$-type modulation doping have recently been examined by a number of groups and have generated much interest due to reports of a temperature insensitive threshold current and increased modulation bandwidth. ${ }^{7-9}$ The modeling of $p$-doped quantum dot structures, which predicts the reduced threshold current and improved modulation response due to an increased peak modal gain and differential gain, ${ }^{10}$ has been reported, and this modeling is consistent with experimentally observed data including laser wavelength as a function of cavity length. ${ }^{11}$ However, the threshold current density measured in most of these $p$-doped laser structures (e.g., Refs. 8 and 9) appears to be higher than those in corresponding undoped structures and the mechanisms by which $p$ doping affects the threshold current density are a matter of current debate.

In this paper we return to the issue of how $p$ doping produces a beneficial effect in $1.3 \mu \mathrm{m}$ emitting quantum dot lasers, show direct measurements of an enhancement in the modal gain in $p$-doped structures, but also show that the threshold current density can still be increased over nominally undoped devices. We begin by examining how the asymmetry in the movement of the quasi-Fermi levels affects the probability of occupation of electron and hole states, and therefore the gain, in a typical quantum dot laser structure 
and in a corresponding $p$-doped structure using a simple model based on a thermal distribution of carriers in the available states. We show that both the wetting layer and excited dot states play a role in the nonideal performance of $1.3 \mu \mathrm{m}$ emitting quantum dot structures. We go on to report direct measurements of the peak modal gain as a function of drive current density and quasi-Fermi level separation that demonstrate the qualitative and quantitative improvements in gain for increasing levels of $p$ doping.

\section{CALCULATIONS}

To understand our experimental results we will first calculate the form of the degree of inversion of the available dot states, which is related to the optical gain, as a function of the quasi-Fermi level separation in a $1.3 \mu \mathrm{m}$ quantum dot laser structure. In doing so we are assuming that the electrons and holes in the dot structure are separately distributed according to global Fermi-Dirac statistics, which is the case in at least some dot structures. ${ }^{12}$ The gain at photon energy $h \nu$ due to a single dot can be written in the form ${ }^{10,13}$

$$
G(h \nu)=G^{\max }(h \nu)\left[f_{c}\left(E_{1}\right)-f_{v}\left(E_{2}\right)\right],
$$

where $f_{c}$ and $f_{v}$ are the electron occupation probabilities for conduction and valence states within a dot, which are separated by the energy $E_{1}-E_{2}=h \nu . G^{\text {max }}$ is a combination of terms including the overlap of the electron and hole wave functions with the optical mode and the matrix element. The gain is directly proportional to $f_{c}-f_{v}$ (the difference in occupation probability of electron and hole states or degree of inversion), with a value of $f_{c}-f_{v}$ of greater than 0 implying positive gain and a value of 1 implying full inversion. Equation (1) is modified for an ensemble of dots in the presence of significant homogenous broadening, but the gain that can be obtained is still related to $f_{c}-f_{v}$ and increases with increasing $f_{c}-f_{v}$.

$p$ doping could change the value of $G^{\max }$ through electrostatically induced changes in the electron and hole wave function overlap and through changes in the homogenous broadening. However, as will now be described, we believe these effects are relatively small in our samples compared to the $p$-dopant dependent change in the degree of inversion. The results of measurements of the photocurrent absorption spectrum as a function of applied bias, where both the magnitude of absorption and energy of the peak of the absorption spectrum change, have been interpreted as evidence of a change in the electron and hole wave function overlap due to the quantum confined Stark effect. ${ }^{14,15}$ However, in such measurements with our samples we observe no change in the energy of the peak of the absorption spectrum as a function of applied bias ${ }^{16}$ indicating that the overlap of the electron and hole wave functions is insensitive to the local electric field in our samples. The modeling of quantum well structures, for example, ${ }^{17}$ based on the self-consistent solution of the Schrodinger-Poisson equations and with carrier density dependent broadening of the Fermi golden rule optical gain spectrum, suggests that the electrostatic deformation of energy bands (here described as a relative movement of the quasi-Fermi levels and included within $f_{c}-f_{v}$ ) has a much larger effect than the carrier density dependent homogenous broadening. Therefore we will focus here on changes in the degree of inversion, and to understand the effect of $p$ doping we will simulate the behavior of $f_{c}-f_{v}$ in our structures with and without $p$ doping.

To calculate the probability of occupancy of the conduction $\left(f_{c}\right)$ and valence $\left(f_{v}\right)$ states corresponding to an energy separation of $0.954 \mathrm{eV}$ (wavelength $=1.3 \mu \mathrm{m}$ ) we first calculate the distribution of states within the dot structure and then populate the electron and hole states according to FermiDirac statistics with charge neutrality over all the states in the system. We make use of experimental spontaneous emission data ${ }^{18}$ for one of our typical $1.3 \mu \mathrm{m}$ emitting quantum dot laser structures, which gives us the dot transition energies of $0.956,1.022,1.093$, and $1.160 \mathrm{eV}$ for ground and excited states, respectively, and the wetting layer transition energy of $1.260 \mathrm{eV}$. These data are consistent with harmonic oscillator forms of the quantum dot conduction and valence potentials where the resulting allowed dot electron and hole states are equally spaced, resulting in equally spaced, transition energies with energy spacing of $67 \pm 4 \mathrm{meV}$. The relative positions (in energy) of the dot and wetting layer states are calculated assuming a conduction band/valence band offset ratio of $80 / 20$ (Ref. 13) and using conduction (0.023 of the free electron mass $\left.m_{0}\right)$ and valence $\left(0.39 m_{0}\right)$ masses taken from the literature ${ }^{19}$ for unstrained bulk InAs, assuming no intermixing of the deposited material. The resulting electron and hole states are separated by 59 and $8 \mathrm{meV}$, respectively. In addition to the single wetting layer transition energy there are higher lying quantum-well-like transitions due to the $\mathrm{In}_{0.15} \mathrm{Ga}_{0.85} \mathrm{As}$ quantum well in which the dots and wetting layer form. We calculate energy levels in the $\operatorname{In}_{0.15} \mathrm{Ga}_{0.85} \mathrm{As}$ strained quantum well with GaAs barriers. For the density of states we use in-plane hole masses derived from a simple 2 $\times 2 k \cdot p$ calculation for this quantum well and also use the electron mass and the relatively light heavy hole mass (of the strained $\mathrm{In}_{0.15} \mathrm{Ga}_{0.85} \mathrm{As}$ ) for the wetting layer levels, where we are uncertain of the composition of the material. The ground and excited electron and hole states due to the 3 $\times 10^{10}$ dots $\mathrm{cm}^{-2}$ are broadened using Gaussian functions. The dot density of $3 \times 10^{10} \mathrm{~cm}^{-2}$ is based on atomic force microscopy (AFM) measurements on uncapped test structures. The heavier hole mass produces a Gaussian distribution of states that is much narrower than that of the electron states with a total transition energy distribution set to agree with that taken from fitting Gaussian functions $(\sigma$ $=30 \mathrm{meV})$ (Ref. 20) to measured absorption data. The states we have calculated are represented in Fig. 1 where it is clear that there are many more wetting layer and quantum well states than dot states by more than three orders of magnitude.

The energy position of the electron and hole quasi-Fermi levels to achieve charge neutrality over the states described above are calculated as a function of the quasi-Fermi level separation and the probability of occupation of conduction and valence states (with electrons), $f_{c}$ and $f_{v}$, with an energy separation corresponding to $1.3 \mu \mathrm{m}$ obtained. The individual values of $f_{c}$ and $f_{v}$ and $f_{c}-f_{v}$ are plotted as the solid lines in Fig. 2. This confirms that the relatively closely spaced dot valence states and a relatively large number of wetting layer 


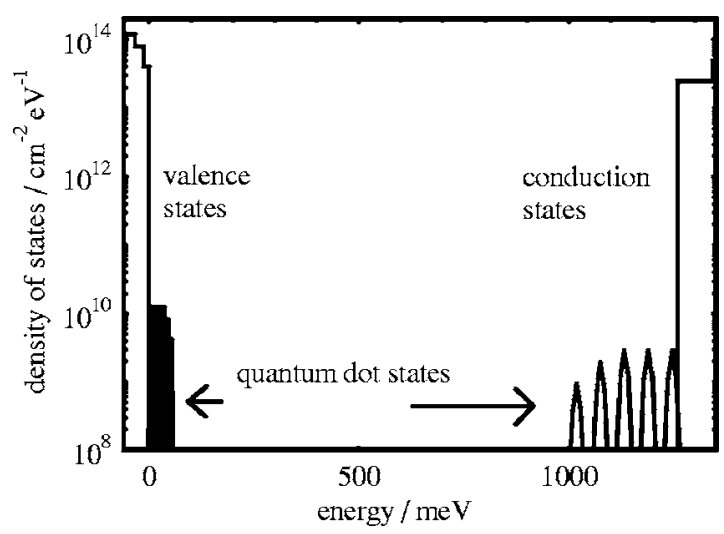

FIG. 1. Calculated states for the strained $\operatorname{In}_{0.15} \mathrm{Ga}_{0.85}$ As quantum well, wetting layer, and the ground and excited dot states where the relative positions in energy are derived from experimental absorption data for the transition energies, literature value of the masses, and assuming a conduction band valence band offset ratio of $80 / 20$.

states damp the movement of the Fermi energy and so complete population inversion $\left(f_{c}-f_{v}=1\right)$ cannot be achieved within the dots even at the highest quasi-Fermi level separation shown here, which corresponds to carrier densities approximately 11 times greater than the quantum dot density. At the highest quasi-Fermi level the conduction states are almost completely populated and the problem is due to the incomplete removal of electrons from the valence states.

There is some uncertainty in the values of electron and hole masses used for the quantum dots since the material may be to some extent strained and/or interdiffused, and in either of these cases the electron and hole masses will be more similar than the values described above. To treat the most severe case we can imagine we have therefore performed the calculation a second time using equal electron and hole dot masses $\left(0.023\right.$ of the free electron mass $\left.m_{0}\right)$, which means that the dot electron and hole states are equally spaced. In addition this has the benefit of allowing us to see just the effect of the wetting layer and quantum well on the relative movement of the quasi-Fermi levels. These results are plotted as the dashed lines in Fig. 2 where we see that even without the effect of the closely spaced dot hole states

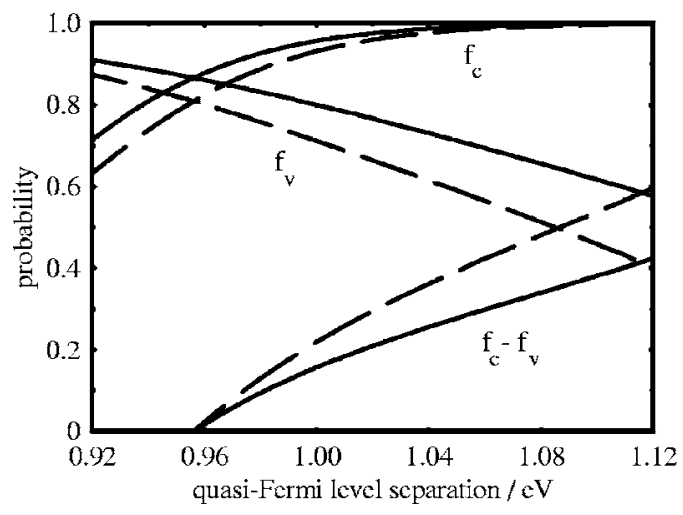

FIG. 2. Probability of occupation of a conduction state and a valence state with an electron for a pair of states corresponding to $1.3 \mu \mathrm{m}$ and the difference between the probabilities $f_{c}-f_{v}$, which indicates the degree of inversion for an undoped structure at $300 \mathrm{~K}$. The calculation of electron and hole dot states used either effective masses for InAs (solid lines) or equal electron and hole masses (dashed lines).
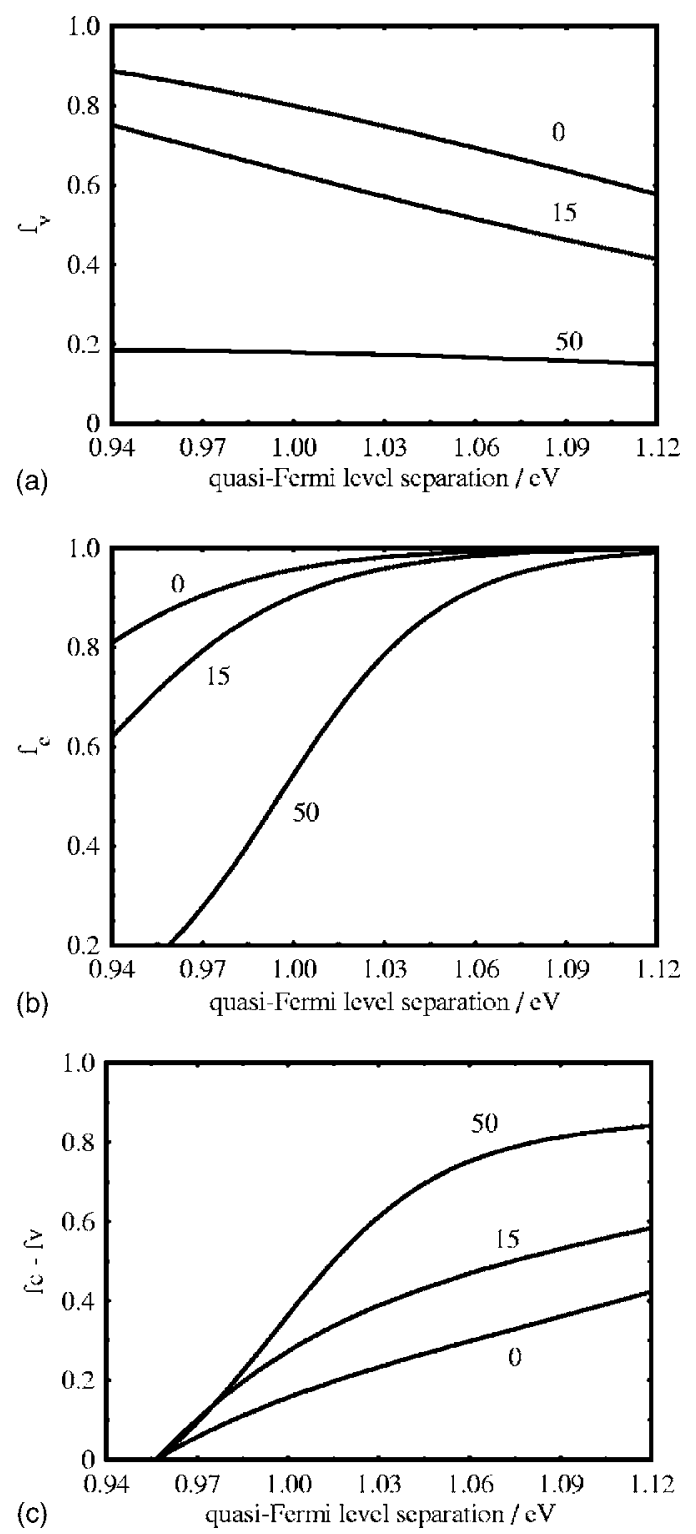

FIG. 3. (a) Calculated probability of occupation of a valence state by an electron for an undoped structure and where an extra 15 or 50 holes per dot are added to the system, and (b) the probability of occupation of the conduction state with an electron under the same conditions and (c) the resultant $f_{c}-f_{v}$ for the undoped sample and in the case where there are an extra 15 or 50 holes per dot.

the probability of occupation of an electron in the valence state corresponding to $1.3 \mu \mathrm{m}$ emission is still too high and that the inversion factor $\left(f_{c}-f_{v}\right)$ is still consequently below the maximum value.

To investigate the role of $p$ doping we simply allow overall charge neutrality to be achieved with an additional number of holes in the system. In Figs. 3(a) and 3(b) we see the effect of adding an extra 15 or 50 holes per dot on the probability of occupation of the valence and conduction states, respectively. The probability of occupation of a valence state with an electron decreases at a fixed quasi-Fermi level separation as extra holes are added to the system while the corresponding probability of occupation of the conduction state with an electron also decreases. Providing that the decrease in the probability of occupation of the valence state is larger than the decrease in the probability of occupation of 


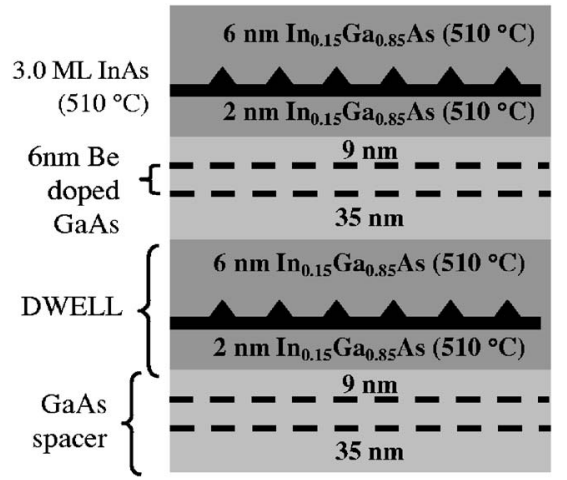

FIG. 4. Schematic representation of two repeats of the five layer DWELL structure indicating the position of the $p$ doping. The growth direction is from the bottom to the top.

a conduction state the overall gain [proportional to $f_{c}-f_{v}$ shown in Fig. 3(c)] will increase with increasing levels of $p$ doping.

\section{EXPERIMENTAL DEVICE STRUCTURES}

We take measurements on three $1.3 \mu \mathrm{m}$ emitting laser structures that are nominally identical except for the $p$ doping. The active region consists of five layers of InAs dots each grown in an $\operatorname{In}_{0.15} \mathrm{Ga}_{0.85}$ As quantum well to form a dots in well (DWELL) structure within a GaAs/ $\mathrm{Al}_{0.40} \mathrm{Ga}_{0.60} \mathrm{As}$ core/clad waveguide. Each DWELL layer is separated from the next by $50 \mathrm{~nm}$ of GaAs and within $6 \mathrm{~nm}$ of this GaAs, placed $9 \mathrm{~nm}$ from the previous D-WELL, is the $p$ doping. This structure is represented schematically in Fig. 4. The nominal doping levels used are $0,7.5 \times 10^{17}$, and 2.5 $\times 10^{18} \mathrm{~cm}^{-3}$ which correspond to approximately 0,15 , and $50 \mathrm{Be}$ atoms per dot. The samples are fabricated into $50 \mu \mathrm{m}$ wide oxide isolated stripe lasers and multisection devices with uncoated facets and are operated pulsed with a pulse length of $400 \mathrm{~ns}$ and a duty cycle of $0.04 \%$ to avoid selfheating.

\section{EXPERIMENTAL METHOD AND RESULTS}

We wish to examine the effect of $p$ doping on the samples and so our first measurements are to determine whether the samples that are nominally identical except for the level of $p$ doping really are identical. We perform measurements of the optical loss (absorption and internal optical mode loss) using the multisection method ${ }^{21}$ under reverse bias. The reverse bias is necessary to remove holes from the structure, provided by the dopants, that are otherwise captured by the dots and block the measurement of absorption that reflect the states that are present in the structure. ${ }^{22} \mathrm{~A}$ reverse bias of $2 \mathrm{~V}$ was applied for all the samples as it was observed that the measured absorption saturates at and above this voltage for all the structures. The modal loss spectrum for each sample is shown in Fig. 5. The region where each curve goes flat at low photon energies gives the waveguide loss of the sample and this is the same for each sample, within the uncertainty of the experiment, at $2 \pm 2 \mathrm{~cm}^{-1}$. Similar waveguide losses are obtained without the application of reverse bias indicating that free carrier losses resulting from

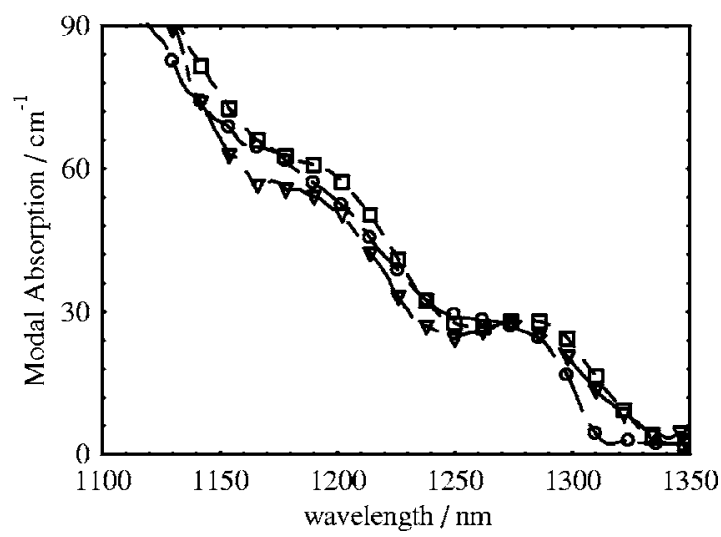

FIG. 5. Measured TE polarized modal absorption spectra at $300 \mathrm{~K}$ for the undoped (circles), $p$ doped with 15 acceptors per dot (squares), and $p$ doped with 50 acceptor atoms per dot (triangles). Each spectrum was measured under a reverse bias of $2 \mathrm{~V}$.

the doping are negligible for the doping levels used in the present structures. This is consistent with previous measurements, where the value of $\alpha_{i}$ was inferred from threshold current measurements performed on devices of different lengths, where an increase in waveguide loss was only observed at higher levels of doping than that used here. ${ }^{11}$ All three samples show similar absorption characteristics with ground state absorption occurring at around $0.973 \mathrm{eV}$ and an excited state transition centered around $1.040 \mathrm{eV}$. The strength of the absorption is also approximately the same for each sample. This suggests that the presence of the acceptor ions has not significantly affected the electronic states within the samples, even though previous work has suggested that the presence of free carriers during growth can affect the interdiffusion of the dots and surrounding material. ${ }^{23}$

Having established that the samples are extremely similar except for the level of $p$ doping we use the multisegment method $^{21}$ to measure the net modal gain spectra as a function of drive current density. A set of spectra with transverse electric (TE) polarization is shown in Fig. 6 for the sample doped with 50 atoms per dot. The transverse magnetic (TM) polarized emission from the structures is too small to measure. These spectra are typical of those from all three samples with

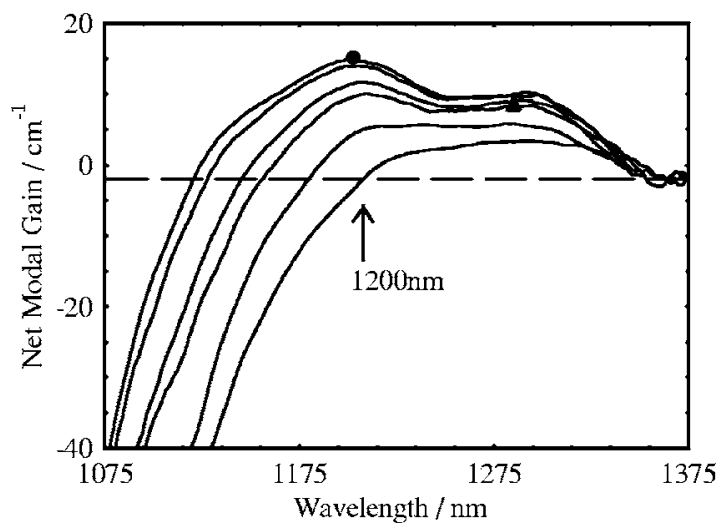

FIG. 6. Net modal gain spectra measured at $300 \mathrm{~K}$ as a function of drive current density between 160 and $880 \mathrm{~A} \mathrm{~cm}^{-2}$ for the undoped sample. The measured value of internal optical mode loss is marked by the dashed line and the transparency point is indicated for the $160 \mathrm{~A} \mathrm{~cm}^{-2}$ data. 


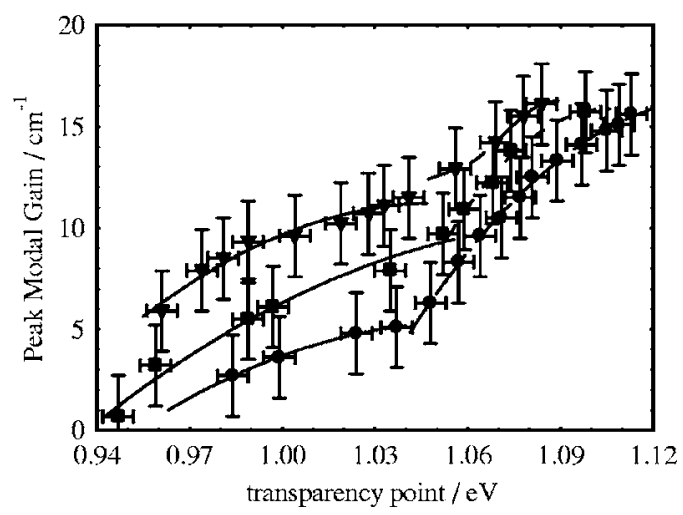

FIG. 7. Peak modal gain plotted as a function of transparency point at $300 \mathrm{~K}$ for the undoped (circles), $p$ doped with 15 acceptors per dot (squares), and $p$ doped with 50 acceptors per dot (triangles). The solid line is a guide to the eye that indicates that the peak gain is obtained from the ground state, while the dashed lines indicate that the gain is from the excited state.

two obvious broad peaks in the spectra, which correspond to the inhomogenously broadened ground and excited states at $\sim 1300$ and $1215 \mathrm{~nm}$. The net modal gain $G-\alpha_{i}$ of Fig. 6 is the gain available to overcome the mirror losses $1 / L \ln (1 / R)$ in a laser of length $L$ and with facet reflectivity $R$. To give some appreciation of the level of gain being achieved in this $p$-doped material the mirror loss of two uncoated facet lasers of lengths of 1000 and $2000 \mu \mathrm{m}$ are plotted versus the lasing wavelength as the points in Fig. 6. Ground state operation is achieved for the longer device. When comparing the magnitude of gain with results reported elsewhere it should be noted that this level of gain is being achieved in a low confinement structure (with a low $\mathrm{Al}$ content $\mathrm{Al}_{0.4} \mathrm{Ga}_{0.6} \mathrm{As}$ cladding) which also has high conductivity and is appropriate for cw performance.

As described earlier the $p$ doping is thought to shift both quasi-Fermi levels towards the valence states which, providing that the increase in valence state occupation probability is larger than the decrease in conduction state probability, will lead to an increase in the gain obtained at a fixed quasiFermi level separation. Experimentally we can obtain the transparency point, defined as the point when the gain equals the losses, i.e., when $G-\alpha_{i}=-\alpha_{i}$, from the measured net modal gain spectra (e.g., approximately $1200 \mathrm{~nm}$ for the lowest current spectrum of Fig. 6). If the carriers can be described by Fermi-Dirac statistics the transparency point corresponds to the quasi-Fermi level separation. We can therefore examine how the gain varies for different levels of $p$ doping at a fixed quasi-Fermi level separation.

Using the measured $G-\alpha_{i}$ spectra and the measured values of $\alpha_{i}$ we derive the values of the peak modal gain and plot these versus the transparency point for the three samples in Fig. 7. Most gain is obtained at any fixed transparency point for the sample with 50 acceptors per dot (triangles), with the sample with 15 acceptors per dot (squares) producing more gain than the undoped sample (circles). The lines associated with each set of data in Fig. 7 indicate when the gain is from the ground state (solid lines) and from the excited state (dashed lines). It can be seen that the introduction of dopant has led to an increase in the transparency point at

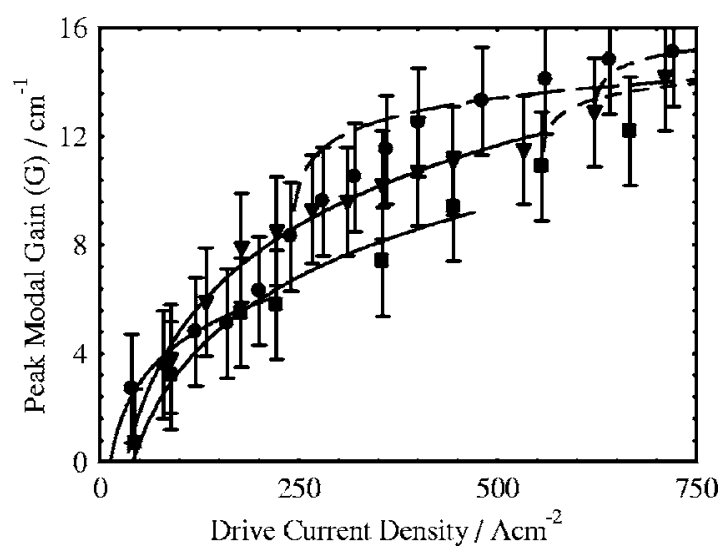

FIG. 8. Peak modal gain plotted as a function of drive current density at $300 \mathrm{~K}$ for the undoped (circles), $p$ doped with 15 acceptors per dot (squares), and $p$ doped with 50 acceptors per dot (triangles). The solid line indicates that the peak gain is from the ground state, while the dashed line shows where the peak gain is from the excited state.

which more gain is obtained from the excited state than the ground state. For the sample without doping (circles) this occurs at $1.040 \pm 0.003 \mathrm{eV}$, while with doping it increases to $1.055 \pm 0.003$ and $1.053 \pm 0.003 \mathrm{eV}$ for 15 (squares) and 50 (triangles) dopants per quantum dot, respectively. This increase in transparency point shows that the samples can be operated up to higher drive levels on the ground state, when doping is present.

The increase in gain with increasing level of doping when plotted as a function of the transparency point is of a very similar form to the results of the calculation shown in Fig. 3(c) and suggests that $p$ doping is indeed enhancing the gain by reducing the asymmetry in the movement of the quasi-Fermi levels. Having understood this behavior we turn to the gain obtained as a function of current density. In Fig. 8 the peak gain-current density curves are plotted for each sample at $300 \mathrm{~K}$. Within the error of the measurement the three samples all have very similar curves, and at the highest drive currents the undoped sample seems to provide the most gain in apparent contradiction to the results of Fig. 7. The solid lines again indicate that the peak gain is from the ground state, while the dashed lines indicate the gain is from the excited state. The apparently anomalous higher gain that is obtained for the undoped sample at high current densities occurs because the peak gain switches to the excited state, with its larger degeneracy and larger available gain, at a lower current density for this sample. Of course, this is not useful when operation at a wavelength of $1.3 \mu \mathrm{m}$ is required and, in fact, there is a significant advantage in the increase in the current density required before the peak gain from the excited state becomes larger than the peak gain from the ground state when using $p$ doping. This is again consistent with the understanding gained from the simple model where the excited electron energy states are relatively depopulated by the shift of the quasi-Fermi levels towards the valence states in the $p$-doped samples.

In the region where all the devices are operating on the ground state (under $250 \mathrm{~A} \mathrm{~cm}^{-2}$ ) the peak modal gains are similar for the three doping levels, with only a small extra gain $\left(\sim 2 \mathrm{~cm}^{-1}\right)$, which is less than the experimental uncertainty, for the highest doped sample. 


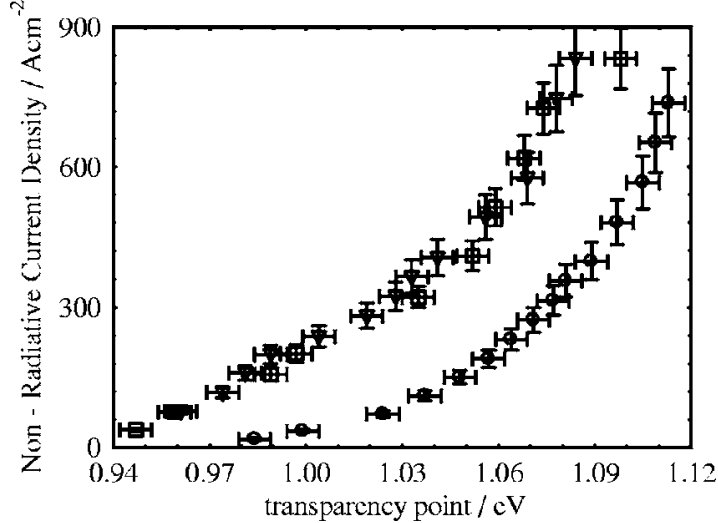

FIG. 9. Nonradiative current density as a function of the transparency point at $300 \mathrm{~K}$ for the undoped (circles), $p$ doped with 15 acceptors per dot (squares), and $p$ doped with 50 acceptors per dot (triangles).

To discover the reason for this we have determined the nonradiative current density as a function of the transparency point for each sample. The nonradiative current density is calculated by removing the spontaneous current density, which is also derived from the multisection experiment, ${ }^{21}$ from the total current density (stimulated emission is negligible ${ }^{21}$ in this segmented contact experiment). The data are plotted in Fig. 9, where it is clear that both the doped samples have a higher value of nonradiative current at any fixed value of transparency point than the undoped material. Therefore although more gain is available at a fixed transparency point (quasi-Fermi level separation) for the doped samples, due to increased nonradiative current paths, more current is needed to reach the same quasi-Fermi level separation so that all three samples have similar gain-current characteristics. A reduced nonradiative lifetime in $p$-doped samples has previously been reported ${ }^{24}$ in time resolved photoluminescence studies and it has been suggested that Auger recombination may be more significant in $p$-doped structures. $^{8,25}$ However, since our results show that the gain at a fixed transparency point increases monotonically with increased doping but the non radiative current density is similar for both 15 and 50 acceptors per dot it may be possible to find structures where benefits of increased gain outweigh the disadvantage of increased nonradiative recombination and the threshold current density is reduced by $p$ doping. To this end we examined a further pair of samples with a similar design to those described earlier. In this case the samples contain either 0 or 18 dopant atoms per dot and there are seven repeats of the DWELL layers rather than five in each sample. In all other respects the structures are nominally identical and we again confirmed that the states in the structure are similar by measuring the absorption. Following the same approach described above we plot in Fig. 10 the peak modal gain as a function of current density and in Fig. 11 the nonradiative current density as a function of the transparency point. In Fig. 11 the nonradiative recombination is much more similar for the doped and undoped samples, which means that a particular value of peak gain can be achieved at a lower total current density as is clear from Fig. 10. If Auger recombination is more significant in $p$-doped samples it is clear that other nonradiative mechanisms must

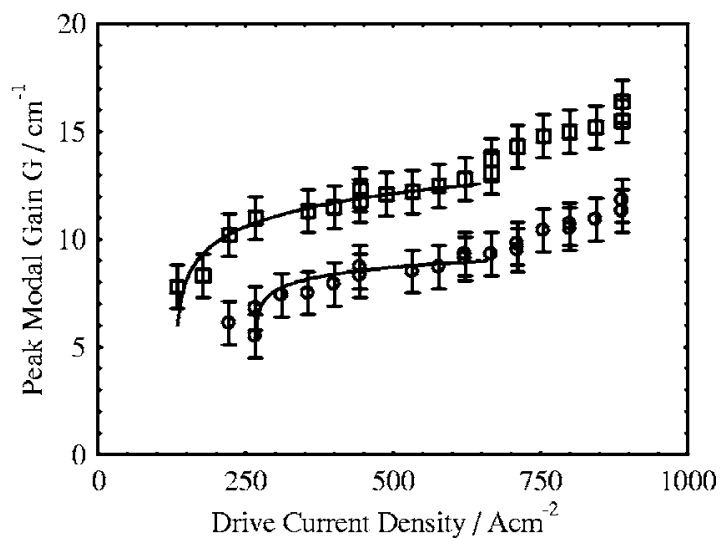

FIG. 10. Peak modal gain plotted as a function of drive current density at $300 \mathrm{~K}$ for the seven DWELL layer samples that are undoped (circles) and $p$ doped at a level of 18 acceptors per dot (squares). The solid line indicates that the peak gain is from the ground state.

play the more significant role for both the structures of Fig. 11, where doped and undoped samples have similar levels of nonradiative recombination, and the samples of Fig. 9, where changing the level of $p$ doping between 15 and 50 atoms per dot has an insignificant affect on the level of nonradiative recombination.

\section{CONCLUSIONS}

In summary we have performed modal gain and spontaneous emission measurements on both $p$-doped and undoped quantum dot devices (that were otherwise identical). We have shown that the available gain at a given quasi-Fermi level separation increases with the presence of doping. This can be explained on the basis of a quasithermal equilibrium model of the population of the available states with the requirement for overall charge neutrality.

We also observe that with the presence of doping the devices can be driven harder before the gain switches from the ground to the excited state. We have shown that the improvement in the gain-Fermi level relationship can be offset or negated by an increase in nonradiative current in the doped samples resulting in similar gain-current density results for both doped and undoped samples. However, it is

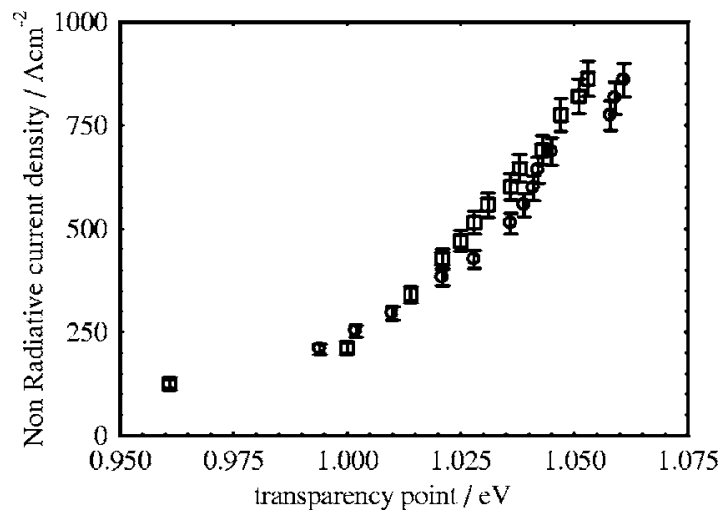

FIG. 11. Nonradiative current density as a function of the transparency point at $300 \mathrm{~K}$ for the seven DWELL layer samples that are undoped (circles) and $p$ doped with 18 acceptors per dot (squares). 
also possible for the benefits due to an increased gain to outweigh the increase in nonradiative recombination in the p-doped samples.

\section{ACKNOWLEDGMENT}

The authors acknowledge the financial support of the UK EPSRC under Grant No. GR/S49308/01.

${ }^{1}$ E. Yablonovitch and E. O. Kane, J. Lightwave Technol. 4, 504 (1986).

${ }^{2}$ N. Chand, E. E. Becker, J. P. Van der Ziel, S. N. G. Chu, and N. K. Dutta, Appl. Phys. Lett. 58, 1704 (1991).

${ }^{3}$ G. W. Turner, H. K. Choi, and M. J. Manfra, Appl. Phys. Lett. 72, 876 (1998).

${ }^{4}$ K. J. Vahala and C. E. Zah, Appl. Phys. Lett. 52, 1945 (1988).

${ }^{5}$ T. Takahashi and Y. Arakawa, Optoelectron., Devices Technol. 3, 155 (1988).

${ }^{6}$ O. B. Shchekin and D. G. Deppe, Appl. Phys. Lett. 80, 2758 (2002).

${ }^{7}$ K. Otsubo et al., Jpn. J. Appl. Phys., Part 2 43, L1124 (2004).

${ }^{8}$ S. Fathpour, Z. Mi, P. Bhattacharya, A. R. Kovsh, S. S. Mikhrin, I. L. Krestnikov, A. V. Kozhukov, and N. N. Ledentsov, Appl. Phys. Lett. 85 , 5164 (2004).

${ }^{9}$ S. S. Mikhrin et al., Semicond. Sci. Technol. 20, 340 (2005).

${ }^{10}$ D. G. Deppe, H. Huang, and O. B. Shchekin, IEEE J. Quantum Electron. 38, 1587 (2002).

${ }^{11}$ O. B. Shchekin and D. G. Deppe, Appl. Phys. Lett. 80, 3277 (2002).
${ }^{12}$ H. D. Summers, J. D. Thomson, P. M. Smowton, P. Blood, and M. Hopkinson, Semicond. Sci. Technol. 16, 140 (2001).

${ }^{13}$ A. A. Dikshit and J. M. Pikal, IEEE J. Quantum Electron. 40, 105 (2004).

${ }^{14}$ X. Huang, A. Stintz, H. Li, A. Rice, G. T. Liu, L. F. Lester, J. Cheng, and K. J. Malloy, IEEE J. Quantum Electron. 37, 414 (2001).

${ }^{15}$ P. W. Fry, I. E. Itskevich, D. J. Mowbray, M. S. Skolnick, J. J. Finley, J. A. Barker, E. P. O'Reilly, L. R. Wilson, I. A. Larkin, and P. A. Maksym, Phys. Rev. Lett. 84, 733 (2000).

${ }^{16}$ I. C. Sandall, C. L. Walker, P. M. Smowton, D. J. Mowbray, H. Y. Liu, and M. Hopkinson, IEE Proc.: Optoelectron. 153, 316 (2006).

${ }^{17} \mathrm{~S}$. Seki, K. Yokoyama, and P. Sotirelis, IEEE J. Sel. Top. Quantum Electron. 1, 264 (1995)

${ }^{18}$ I. C. Sandall, P. M. Smowton, C. L. Walker, H. Y. Liu, M. Hopkinson, and D. J. Mowbray, IEEE Photonics Technol. Lett. 18, 965 (2006).

${ }^{19}$ M. P. C. M. Krijn, Semicond. Sci. Technol. 6, 27 (1991).

${ }^{20}$ P. M. Smowton, C. L. Walker, I. C. Sandall, I. R. Sellers, D. J. Mowbray, H. Y. Liu, M. Gutierrez, and M. Hopkinson, Proc. SPIE 5738, 332 (2005).

${ }^{21}$ P. Blood, G. M. Lewis, P. M. Smowton, H. D. Summers, J. D. Thomson, and J. Lutti, IEEE J. Sel. Top. Quantum Electron. 9, 1275 (2003).

${ }^{22}$ I. C. Sandall, P. M. Smowton, C. L. Walker, T. Badcock, D. J. Mowbray, H. Y. Liu, and M. Hopkinson, Appl. Phys. Lett. 88, 111113 (2006).

${ }^{23}$ O. B. Shchekin, D. G. Deppe, and D. Lu, Appl. Phys. Lett. 78, 3115 (2001).

${ }^{24}$ J. Siegert, S. Marcinkevicius, and Q. X. Zhao, Phys. Rev. B 72, 085316 (2005).

${ }^{25}$ S. Mokkapati, M. Buda, H. H. Tan, and C. Jagadish, Appl. Phys. Lett. 88, 161121 (2006) 